\title{
Erfahrungen mit Tuberkulin Rosenbach bei Lungentuberkulosen.
}

\author{
Von
}

\section{Martin Bischel,}

Medizinalpraktikant.

Im Jahre 1910 gab Friedrich J. Rosenbach ein neues Tuberkulinpräparat an, bei dem durch die Einwirkung eines Trichophytonpilzes die giftigen unverträglichen Stoffe gegenüber den wirksamen Bestandteilen stärker zurücktreten sollen, als dies bei anderen Tuberkulinen der Fall ist. Tuberkulin Rosenbach soll an Toxizität etwa $100 \mathrm{mal}$ schwächer sein als Alttuberkulin Koch. Es bietet daher den Vorteil der Anwendung in bedeutend höheren Dosen.

Im Laufe der 4 Jahre sind nun eine Reihe von Arbeiten über das neue Tuberkulin erschienen. Die Mehrzahl der Autoren kommt zu einer günstigen Beurteilung.

Am hiesigen Landkrankenhause wird Tuberkulin Rosenbach seit Mai 1912 verwendet; sowohl bei Lungentuberkulose als auch bei chirurgischer Tuberkulose. Ich möchte nur auf die Erfahrungen, die wir bei Lungentuberkulose machten, näher eingehen. Behandelt wurden 46 Fälle verschiedener Stadien, doch wurden inzipiente Formen bevorzugt. 21 Fälle, bei denen eine längere Beobachtung möglich war, sind weiter unten im Auszug wiedergegeben, die anderen Faille waren entweder zu kurze Zeit in Behandlung, um ein Urteil gewinnen zu können, oder es lagen Komplikationen vor.

Die Injektionen wurden am Oberarm subkutan vorgenommen. In den meisten Fällen wurde das Tuberkulin unverdünnt injiziert. Bei sehr kleinen Dosen wurde mit physiologischer Kochsalzlösung 
verdünnt. Als Anfangsdosis wurde immer 0,1 verabreicht, traten wie bei der Mehrzahl der Fälle keine starken Reaktionen auf, so wurde die Dosis um je 1 Dezigramm gesteigert bis zur Enddosis 1,5 (in vereinzelten Fällen höher). Die Injektionen wurden 2 mal wöchentlich vorgenommen. Traten stärkere Lokal- oder Allgemeinreaktionen auf, so wurde die gleiche Dosis mehrmals wiederholt bis zur Gewöhnung, oder es wurde auf niedere Dosen heruntergegangen.

Ich lasse zunächst die Krankengeschichten im Auszug folgen.

Fall 1. Journal-Nr. 791, 1912; weiblich, 24 Jahre alt; behandelt vom 18. Juni 1912 bis 5. März 1913.

Anamnese: Vater, 1 Broder an Lungentuberkulose gestorben. Patientin hatte im Winter 1911 Pleuritis sicca, seit $3 / 4$ Jahr leidet Patientin an Stechen in der linken Schulter, Husten und Auswurf; bisweilen Nachtschweisse.

Status: 24jähriges junges Mädchen, guter Ernährungszustand, Thorax flach, Halsdrüsen links geschwollen.

Pulmones: Grenzen normal. R.H.O. Schallverkürzung, Vesiko-Bronchialatmen, vereinzelte Rasselgeräusche.

L.H.O. Schallverkürzung bis $3 \mathrm{Bw}$., Vesiko-Bronchialatmen.

Mittelblasige feuchte Rasselgeräusche.

Tbc. nachgewiesen. Sonst normaler Organbefund.

Diagnose: Tuberkulose beider Lungen.

Beginn der Behandlung mit Tuberkulin Rosenbach am 1. Juli.

9. September. Patientin hat geit jhrer Aufnahme 10 Pfd. zugenommen; die Schmerzen haben nachgelassen, Husten und Auswurf dagegen in gleicher Weise wie früher vorhanden.

12. Dezember R.H.O. bis 5 Bw. R.V.O. bis Klv. Dämpfung; verschärftes Atemgeräusch, Rasselgeränsche. L.H.O. bis 6 Bw. L.V.O. bis 2. Rippe Dämpfung, Bronchialatmen, feuchtes Rasseln. Tuberkelbazillen nachgewiesen.

März 1913 keine Anderung im Befund. Patientin wird anf ihren Wunsch entlassen.

Es wurden folgende Tuberkulinkuren vorgenommen:

1. 1. VIII. bis 18. IX. Anfangsdosis 0,1, Enddosis 2,0, Gesamtdosis 34,7,

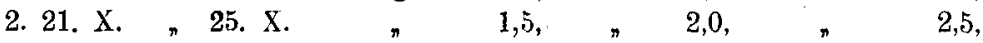

3. 14 XI. $\pi$ 20. I. $\quad$ \% $0,5, \quad, 2,0, \quad n \quad 23$,

4. 14. bis 27. II. $\quad 0,1, \quad n \quad 0,5, \quad$ n 1.5 .

Es wurden also im ganzen $64,7 \mathrm{ccm}$ verabreicht.

Gewichtsverhältnisse: Gewicht bei der Aufnahme 116, Höchstgewicht 130 Pfd. Gewicht bei der Entlassung 123 Pfd.

Die Temperatur wurde durch Tuberkulin Ros. entschieden günstig beeinflusst. In der Zeit vom 1. VII. bis 19. VIII. hielten sich die T'emperaturen rektal gemessen auf 36,8 bis 38,3 . Vom 20. VIII. bis 25 . X. wird 38,0 selten erreicht. Vom 25. X. ab beträgt die durchschnittliche Abendtemperatur 37,3 (axillar). Temperaturreaktionen waren nicht vorhanden. Die Injektionen wurden gut vertragen. Thc. wurden immer nachgewiesen. Subjektiv befindet sich Patientin wobler, Husten, Auswurf anverändert. Objektiv Verschlechterung des Lungenbefandes.

Am 15. Mai 1914 kam Patientin wieder zur Aufnahme. Patientin wird in sehr schlechtem Zustand eingeliefert. Lungenbefund: rechte Lunge kompakte 
Dämpfung hinten sowohl wie vorne, L.H.O. Dämpfung bis $6 \mathrm{Bw}$. Linke Spitze Schall verkürzt.

Auskultat.: Über der ganzen rechten Lunge Giemen, Rasseln, Pfeifen, Bronchialatmen mit metallischem Beiklang. L.H.O. Bronchialatmen, Rasseln. Atemfrequenz 32, Temperatur 38-39. Exitus am 1. Juni 1914. Der Fall zeigt also ständige Progredienz.

F all 2. Journal-Nr. 727, 1912; weiblich, 35 Jahre, behandelt vom 9. VI. 1912 bis 20. III. 13.

Anamnese: Fltern gestorben, Todesursache unbekannt. Ein Brader starb an Lungentuberkulose. Patientin hatte vor 4 Jahren Rippenfellentzündung, seit einem Jahr besteht Heiserkeit.

März 1912 Kehlkopfoperation. Patientin klagt jetzt über Husten, Auswarf. Keine Nachtschweisse.

Status: Mittelkräftige Frau, Gewicht 119 Pfd. Brustumfang $84 \mathrm{~cm}$.

Lungen: Grenzen tiefstehend, gut verschieblich.

R.H.O. bis $4 \mathrm{Bw}$. Schallverkürzung, verschärftes bronchiales Inspirium, keine Katarrhalsymptome.

Cor: O. B. Tbc. nachgewiesen.

Diagnose: Tb. pulm. dextr. II.

Es wurden 3 Tuberkulinkuren gemacht.

1. 10. VI. bis 18. IX. Anfangsdosis 0,1, Enddosis 3,0, Gesamtdosis 74,7,

2. 21. X. " 25. X. " " $2,5, \quad " \quad 3,0, \quad \# \quad 8,5$,

3. 14. IX. " 18, I. " " $1,0, \quad$ " $3,0, \quad$ "

Es wurden also in 3 Kuren 131,6 ccm gegeben. Temperaturreaktionen traten nur bei der ersten Kur nach den Dosen 0,4 und 0,5 auf. Die hohe Anfangsdosis 2,5 bei der zweiten Kur wurde ohne jede Reaktion vertragen. Die Temperaturen waren im übrigen während der ganzen Zeit der Behandlung normal.

Befund am 16. XII. R.H.O. bis 6 Bw. Schallverkürzung, bis $4 \mathrm{Bw}$. Dämpfung, Bronchialatmen. R.V.O. bis 2. R. Dämpfung. Bronchialatmen, feuchtes Rasseln. L.H.O. bis 2 Bw. Schallverkürzung, verschärftes Atmen, ebenso linke Spitze. Im Lungenbefund war also ein Fortschreiten des Prozesses auf der rechten Lunge und ein Übergreifen auf die linke Lunge festgestellt. Von Dezember bis März keine Besserung im Befund, anderseits auch kein Fortschreiten. Trotz des aus. gedehnten Lungenbefundes traten keine Temperaturen auf. Das Gewicht betrug bei der Aufnabme 119, bei der Entlassung 129 Pfd. Tbc. wurden immer nachgewiesen.

Nachuntersuchang: Ende August 1913. Patientin ist heiser, klagt über starken Husten, Auswurf, Nachtschweisse, Schmerzen in der linken Seite. Lungenbefund: Schallverkürzung auf der ganzen rechten Lunge, Bronchialatmen, verlängertes bronchiales Exspirium. L.H.O. Schallverkürzung, verschärftes Inspirium. L.V.O. vereinzeltes Knacken. Gewicht 109 Pfd.

Es ergibt sich also eine weitere Verschlechterung des Lungenbefundes und Gewichtsabnahme.

Fall 3. Journal-Nr. 1268, 12; männlich, 26 Jahre alt; behandelt vom 30. XI. 12 bis 19 . IV. 13.

Anamnese: Eltern an Lungenkrankheiten gestorben. Patient leidet seit Dezember 1911 an Husten, Nachtschweissen, Lungenbluten, war 3 Monate in einer Heilstätte. Patient klagt jetzt über Husten, Nachtschweisse, Herzklopfen. 
Status: Mittelgrosser Mann, Gewicht 120 Pfd. Brustkorb flach, hebt and senkt sich mässig beim Atmen. Die rechte Seite schleppt auffallend nach.

Pulmones: Auf der ganzen rechten Seite Schallverkürzung. R.H.O. Dämpfung bis 6 Bw. R.V.O. Dämpfung bis 3. R. L.H.O. Dämpfang bis 1 Bw. L.V.O. Dämpfung bis Klav.

Anskultat.: R.H.O. Lautes Bronchialatmen, vereinzeltes Giemen und Schnurren. R.V.O. Giemen. L.H.O. und L.V.O. verschärftes Atmen obne Nebengeräusche,

Befund an 28. VI.: Patient fühlt sich subjektiv wohl, hat 20 Pfd. zugenommen. Lungenbefund: Schallverkürzung auf der ganzen rechten Lunge, Dämpfung bis $3 \mathrm{Bw}$, Verlängertes Exspirium. Broncho-vesikuläres Atmen. R.V.O. Dämpfung bis Klav. Broncho-vesikuläres Atmen. Knisterrasseln. L.H.O. Dämpfung bis zur Spina scapulae. L.V.O. Dämpfung bis 1. R., verschärftes Atmen.

Die Tuberkulinkur begann am 17. XII. mit der Dosis 0,05, Enddosis 2,0 an 17. III., Gesamtdosis 11,7.

2. Kur vom 31. III. bis 28. VI. Gesamtdosis 12,0. Im ganzen wurden also $32 \mathrm{ccm}$ verabreicht. Gewicht bei der Aufnahme 120 Pfa., bei der Entlassung 140 Pfu. Die Sputummenge blieb annähernd gleich, schwankte zwischen 10 und $20 \mathrm{ccm}$ pro Tag. Tbc. wurden immer nachgewiesen. Im Lungenbefund keine Besserung. Die Temperaturen, die zu Beginn der Behandlung normal waren, erreichten wäbrend der Tuberkulinbehandlung fast immer 37,5 .

Nachuntersuchung: 28. III. 1914. Patient macht einen sehr elenden Eindruck, klagt über starken Husten, Auswurf, Nachtschweisse. Lungenbefund : R.H.O. Scballverkürzung bis $7 \mathrm{Bw}$. L.H.O. Dämpfung bis $3 \mathrm{Bw}$, rechte Spitze gedämpft, R.V.O. Dämpfung bis 3. R.

Auskultat.: R.H.O. Bronchialatmen mit metallischem Beiklang, starke Rasselgeräusche. Rechte Spitze Giemen, Rasseln, verlängertes und verschärftes Exspirium. L.H.O. Rasselgeräusche, pfeifendes Inspirium. Die Nachuntersuchung ergibt also eine weitere Verschlechterung des Lungenbefundes. - Exitus im Juni 1914.

F all 4. Journal-Nr. 880, 13; weiblich, 26 Jahre alt; behandelt vorn 18. VII. 13 bis 29. XI. 13.

Anamnese: Seit 8 Wochen Husten und Stechen in der linken Seite, Fieber, Nachtschweisse, Appetitlosigkeit, starke Abmagerung. Partus: Vor 29 Wochen.

Status: 26jährige Frau, Ernährungszustand scblecht. Schleimhäute anämisch.

Pulmones: L.H.O. Schallverkürzung, verschärftes Atmen. Linker Unterlappen gedämpft. Links in der Axillarlinie deutliches Reiben,

Diagnose: Tb. pulm. sin. Pleuritis.

Beginn der Injektionen mit Tuberkulin Ros. am 24. VII.

Patientin reagierte auf fast alle Injektionen mit Temperatursteigerang bis 38,3, während sonst die höchste Abendtemperatur 37,6 betrug. Schon nach 3 Injektionen (Gesamtdosis 0,6 ) konnte eine Verschlechterung des Lungenbefundes festgestellt werden, die wohl als Herdreaktion zu betrachten ist; es zeigte sich eine Dämpfung über der ganzen linken Lunge mit Bronchialatmen, wenige Tiage später auch Rasseln. Die Injektionen wurden trotz der Temperaturreaktionen fortgesetzt bis zur Enddosis 1,1. Die Gesaintdosis betrug 7,4. Der Lungenbefund verschlechterte sich beständig. 11. IX. linke Lunge völlig gedämpft. L.H $O$. Rasseln, Knacken, Bronchialatmen. L.H.U. Rasseln und Giemen. R.H.O. Schall. verkürzung, Rasseln und Giemen, rechto Spitze gedämpft. Die Injektionen mit Tuberkulin werden jetzt abgebrochen. Patientin klagt über Husten, Stechen und 
Nachtschweisse. Am 29. XI. Entlassung gegen ärztlichen Rat. Im Februar 1914 Exitus. Gewicht bei der Aufnahme 82 Pfd, bei der Entlassung 81 Pfd. Tbc. wurden immer nachgewiesen. Der Fall zeigt eine ständige Progredienz des Lungenbefundes. Auf die ersten Injektionen hin trat eine deutlichere Verschlechterung ein (Herdreaktion).

Fall 5. Journal-Nr. 1246, 13; weiblich, 32 Jahre; behandelt vom $14 . \mathrm{X}$. bis 29. XI. 13 .

Anamnese: Der Mann lungenleidend. Patientin klagt seit dem letzten Partus (vor 18 Wochen) über allgemeine Müdigkeit, Husten, Stechen auf der Brust, seit 4 Wochen zunehmende Verschlimmerung, auch Nachtschweisse.

Status: 32 jährige Frau, Ernährungszustand mässig.

Pulmones: R.H.O. bis $3 \mathrm{Bw}$. Dämpfung, Bronchialatmen, über der ganzen rechten Lunge verschärftes Atmen, R.V.O. Dämpfung bis 2. R. Bronchialatmen. L.H.O. bis $3 \mathrm{Bw}$. Schallverkürzung. L.V. verlängertes broncho-vesikuläres Exspirium.

Diagnose: Tuberkulose beider Lungen.

Befund bei der Entlassung: Keine Nachtschweisse, wenig Husten und Auswurf. Lungenbefund unverändert. Ls wurde eine Spritzkur vorgenommen. Anfangs. dosis 0,1, Enddosis 1,5, Gesamtdosis 12,7. Temperaturen zu Beginn der Behandlung 36,5 bis 37,7. Am Schluss der Behandlung ebenso. Bei der Dosis 0,7 traten cine Temperaturreaktion $(38,3)$ und eine-Stichreaktion auf. Gewicht bei der Aufnahme $99 \mathrm{Pfd}$, bei der Entlassung 104 Pfd. Sputummenge anfangs 10, später $5 \mathrm{ccm}$. Tbc. wurden nicht nachgəwiesen. Nachuntersuchung im Januar 1914: Keine Nachtschweisse, sehr wenig Auswurf, Husten noch vorhanden, Patientin arbeitet. Lungenbefund: R.H.O. Dämpfung bis 5 Bw. L.H.O. ebenso. Rechte Spitze gedämpft, vorne reicht die Dämpfung bis zur 3. R. Auskultat.: R.H.O. Bronchialatmen, R.U. Rasselgeräusche. L.H.O. verschärftes Atmen, linke Spitze verlängertes und verschärftes, fast bronchiales Exspirium. Im Lungenbefund ist also eine weitere Verschlechterung nachweisbar.

Fall 6. Journal-Nr. 161, 13; männlich, 22 Jahre alt; behandelt vom 16. Januar 1913 an.

Anamnese: Mutter an Tuberkulose gestorben, Patient erkrankte 1912 mit Husten und Auswurf. War in der Lungenbeilstätte Berka in Behandlung, dort geheilt entlassen. Im Dezember 1912 wieder Husten, Auswurf, Nachtschweisse.

Status: Mittelgrosser Mann. Ernährungszustand gat, Gesichtsfarbe blass. Thorax mässig gewölbt.

Pulmones: Dämpfung über der rechten Spitzs, R.H.O. Dämpfung bis zur Spina scapulae. Linke Spitze Schallverkürzang.

Auskultat.: R.H.O. Bronchialatmen, Rasselgeräusche. L.H.O. und linke Spitze abgeschwächtes Atmen, Rasseln. Lungengrenzen rechts weniger verschieblich als links.

Diagnose: Tb. pulmonum; Tbc. nachgewiesen. Tuberknlin Rosenbachkuren : 1. Vom 19. I. bis 17. III. Anfangsdosis 0,02, Enddosis 2,0, Gesamtdosis 21,7. 2. Vom 31. III. bis 22. V. Anfangsdosis 0,05, Enddosis 2,0, Gesamtdosis 21,5.

Befund: am 27. V. R.H.O. Dämpfung, Rasseln, R.H. Mitte rauhes Atmen, L.H.O. Schallverkürzung, Rassein.

3. Kur 1. IX. bis 15. X. Anfangsdosis 0,1, Enddosis 1,5, Gesamtdosis 12,0. Es traten wiederholt 'Temperatursteigerungen auf', keine Stichreaktionen.

4. Kur 3. XI. bis 18. XII. Dosierung wie bei der 3. Kur. 
Fast regelmässig Temperaturreaktionen, keine Stichreaktionen. Eine 5. Kur, die im Januar 1914 begonnen wurde, musste wegen einer Hämoptoe $(600 \mathrm{ccm})$ unterbrochen werden. Insgesamt wurden 68,7 cem Tuberkulin injiziert. Am 31. I. erneute Hämoptoe $(700 \mathrm{ccm})$. Der Befund am 27. III. 1914: R.H.O. Schallverkürzung bis $6 \mathrm{Bw}$. R.V.O. Dämpfung bis 2. R.; L.H.O. Schallverkürzung bis $4 \mathrm{Bw}$. Auskultat.: Über der ganzen rechten Lunge starkes Rasseln, in der Höhe des 8 Bw. Bronchialatmen. R.V.O. Rasselgeräusche, Exsp. bronchial mit metal. lischem Beiklang. L.H.O. Rasselgeräusche, verschärftes Insp. Temperatur normal: Husten wenig, keine Nachtschweisse, im Auswurf zahlreiche Tbc. Subjektiv fühlt Patient sich etwas matt, objektiv Verschlechterung des Lungenbefundes.

Fall 7. Journal-Nr. 464, 13; weiblich, 23 Jahre alt; behandelt vom 7 . IV. bis 23. V. 1913.

Anamnese: Patientin erkrankte im Januar mit Erbrechen, Fieber, Schmerzen auf der Brust, Husten, Auswurf und Nachtschweissen. Seit 6 Wochen Verschlimmerung, wiederholt Blut im Auswurf.

Status: Mittelgrosses Mädchen, Ernährungszustand schlecht, Schleimhäuteblass.

Thorax: Schmal, flach, die rechte Hälfte schleppt bei der Atmung. Pulmones: R.H.O. Dämpfung bis 4 Bw. R.V.O. Dämpfung bis 4. R. L.H.U. von 9 Bw. ab Dämpfung. Atemgeränsch R.H.O. rauh, verschärft, R.V.O. verschärft mit verlängertem Exsp., kein Rasseln. Über der Dämpfung L.H.U. abgeschwächtes vesikuläres Atmen.

8. V. Patient fühlt sich wohler, hat an Gewicht zugenommen.

Pulmones: Perkutorisch derselbe Befund wie oben.

Auskultat.: R.H.O. Rasseln, L.H.U. Pleurareiben.

28. V. Auf eigenen Wunsch entlassen. Die Behandlung mit Tuberkulin Ros. begann am 17. IV. mit der Dosis 0,1, Enddosis (am 22. V.) 1,2, Gesamtdosis 6,7. Durchschnittliche Temperatur zu Beginn der Behandlung 37,0-38,2, am Ende der Behandlung 36,8-37,4. Temperaturreaktionen fehlten. Die Gewichtszunahme betrug $10 \mathrm{Pfd}$. Thc. wurden nicht nachgewiesen. Subjektiv fühlt Patientin sich besser, im Lungenbefund geringe Besserung.

Nachuntersuchung: August 1913.

Pulmones: R.H.O. und rechte Spitze Schallverkürzung, vereinzeltes Knacken. L.H.U. Schallverkürzung, Bronchialatmen, Husten gering, sehr wenig Auswurf, keine Nachtschweisse. Subjektives Befinden gut, Lungenbefund annähernd un verändert, geringe Gewichtszunahme.

F a 11 8. Journal-Nr. 914, 13; weiblicb, 16 Jahre; behandelt vom 28. VII. bis 28. IX. 13.

Anamnese: Patientin hatte als Kind Rippenfellentzündung, fühlt sich seit längerer Zeit matt, klagt über Husten; keine Nachtschweisse.

Status: 16 jähriges Mädchen, Ernährungszustand schlecht, Gewicht 69 Pfd. Thorax: Symmetrisch.

Pulmones: L.H.O. bis zur Spina scapulae, Schallverkürzung, Giemen undKnacken. L.V.O. verschärftes Atmen. Tbc. im Sputum und Urin nachgewiesen. Im Urin Eiweiss und Zylinder.

Diagnose: Tb. pulm. sin.; Nephritis. Therapie: Tuberkalin Ros. Nierendiät. Anfangsdosis 0,1, Enddosis 1,5, Gesamtdosis 12,7, Temperaturen durchweg normal. Temperaturreaktionen traten nach der Dosis 0,3 auf. Gewichtszunahme $13 \mathrm{Pfd}$.

Befund am Schluss der Behandlung L.H.O. Giemen, Knacken, etwas verschärftes Atemgeräusch, Husten und Auswurf sehr wenig, keine Nachtschweisse 
subjektives Wohlbefinden, Lungenbefund unverändert. - Patientin kam nicht zur Nachuntersuchung.

F a 11 9. Journal-Nr. 588, 12; männlich, 14 Jahre; behandelt vom 6 . V. bis 16. VIII. 1912.

Anamnese: Patient hatte als Kind geschwollene Drüsen, leidet seit 12 Wochen an Mattigkeit, Husten, Nachtschweissen.

Status: 14 jähriger junger Mensch in leidlich gutem Ernährungszustand.

Pulmones: R.H. auf der ganzen Lunge Schallverkürzung, verlängertes Exsp. Bronchialatmen, zahlreiche Rasselgeräusche. R.V. Schallverkürzung bis $\mathbf{3}$. R. Bronchialatmen, Rasseln. L.H. verschärftes verlängertes Exsp., zahlreiche Rasselgeräusche, ebenso L.V. Tbc. im Sputum nachgewiesen.

Abgangsbefund 15. VIII.: R.H.O. Schallverkürzung bis 6 Bw. Broncho-Vesikuläratmen. R.H.U. verschärftes Insp., vereinzeltes Rasseln. R.V. Schallverkürzang bis 2. R, Broncho-Vesikuläratmen, Rasselgeräusche geschwunden. L.H.O. verlängertes Exsp., keine Rasselgeräusche. Appetit gut, keine Nachtschweisse. Tuberkulinbehandlung vom 9. V. bis 21. VI.: Anfangsdosis 0,1, Enddosis 1,0, Gesamtdosis 6,5. Vom 22. VI. bis 14. VIII.: Anfangsdosis 0,1, Enddosis 2,0, Gesamtdosis $27 \mathrm{ccm}$. Es wurden also im ganzen $33,5 \mathrm{ccm}$ verabreicht. Tempera. turen zu Beginn der Behandlung 37,2 bis 38,5 . Schon am 13. VI. trat Entfieberung ein, Temperatur blieb dawn normal. Temperaturreaktionen traten 2 mal auf. Gewichtszunahme $18 \mathrm{Pfd}$. Subjektives Wohlbefinden, im Lungenbefund Besserung.

Nachuntersuchung: Ende August 1913.

R.H.O. Schallverkürzung bis $6 \mathrm{Bw}$. R.V.O. Schallverkürzung bis 2. R. R.H.O. vereinzeltes Rasseln. L.H.O. verschärftes Insp., sonst normaler Befund. Es zeigt sich also eine weitere Besserung des Lungenbefundes. Gewichtszunahme seit der Entlassung 2 Pfd. Patient wurde mit leichter landwirtschaftlicher Arbeit beschäftigt.

F a 11 10. Journal-Nr. 383, 13; männlich, 18 Jahre; behandelt vom 14. III. 13 bis $9 . \mathrm{V} .13$.

Anamnese: Mutter an Tuberkulose gestorben, Patient erkrankte 1911 an Lungenspitzenkatarrb. Seit einigen Tagen Stechen auf der linken Seite, Husten und Auswurf.

Status: 18 jähriger Mann, Ernährungszustand gut.

Thorax : flach.

Pulmones: Üluer beiden Spitzen Schallverkürzung, besonders über der rechten. R.H.O. Knisterrasse]n. L.H.O. verschärftes Atmen. Tbc. nachgewiesen.

Diagnose: Tb. pulm.; Therapie Tuberkulin Ros.

Befund am 9. V.: Tbn. Ros. wird gut vertragen, Gewichtszunahme 12 Pfd. Übor der rechten Spitze feines Rasseln.

Tuberkulinbehandlung vom 17. III. bis 8. V. Anfangsdosis 0,05, Enddosis 1,6 , Gesamtdosis $9,75 \mathrm{ccm}$.

Temperaturen normal, Temperaturreaktionen traten nicht auf, Gewichtszunahme $12 \mathrm{Pfd}$. Im Lungenbefund Besserung. Tbc. noch nachgewiesen. Sputummenge geringer, zu einer Heilstättenkur entlassen.

Nachuntersuchung: August 1913.

Pulmones: R.H.O. Schallverkürzung bis zar Spina scapulae, rechte Spitze und rechts vorne bis 1 . R. Schallverkürzung. 
Auskultat.: R.H.O. Knacken, Giemen, broncho-vesikuläres Atemgeräusch, Exsp. verlängert, R.V.O. derselbe Befund. Linke Lunge normaler Befund. Husten und Auswurf wenig, keine Nachtschweisse, subjektives Befinden gut.

F a 11 11. Journal-Nr. 243, 13; männlich, 18 Jahre; behandelt vom 5. II. 13 bis 12. IV. 13 .

Anamnese: Mutter an Tuberkulose gestorben, Patient klagt über Mattigkeit, Husten, Auswurf, Nachtschweisse.

Status: Mittelgrosser junger Mensch, Ernährungszustand gut, Thorax flach.

Pulmones: R.H.O. Schallabschwächung bis $3 \mathrm{Bw}$, vereinzeltes Giemen und Brummen. L.H.O. Dämpfung bis $3 \mathrm{Bw}$, linke Spitze Schallverkürzung. Atmung vesiko.bronchial.

Röntgenbild: Beide Spitzen verdunkelt, rechts mehr als links, Zwerchfell gat beweglich.

Befund am 12. IV.: Tbn. Ros. wird gut vertragen.

Pulmones: Linke Spitze Dämpfung, über beiden Spitzen rauhes Atmen, verlängertes Exsp., links auch spärliches Rasseln. Es wurden 13,5 ccm Tbn. Ros. injiziert. Anfangsdosis 0,1, Enddosis 2,0. Temperaturen fast immer normal. Nach der ersten Injektion trat eine Temperaturreaktion auf. Gewichtszunahme $19 \mathrm{Pf}$. Tbc. im Anfang nachgewiesen, am Ende der Behandlung nicht mehr nachweisbar.

Nachuntersuchung: R.H.O. geringe Schallabschwächung, Atemgeräusch broncho-vesikulär, sonst normaler Lungenbefund. Morgens etwas Husten, keine Nachtsehweisse, subjektives Wohlbefinden.

F a 11 12. Journal Nr. 826, 13; männlich, 45 Jahre; behandelt vom 7. VII. 13 bis 2. VIII. 13 .

Anamnese: Mit 22 Jahren Lungenentzündung, im Januar 13 Lungenspitzenkatarrh, Rippenfellentzündung. 9 Wochen in Heilstättenbehandlung. Patient kommt wegen Schluckbeschwerden zur Aufnahme, klagt ausserdem äber Husten und Nachtsehweisse.

Status: Kräftiger Mann, Gewicht 131 Pfd. Die hintere Rachenwand zeigt rechts eine starke Vorwölbung. Unter dem Musc. sternocleido-mast. fühit man einen verschieblichen Tumor. 'Thorax symmetrisch.

Pulmones: R.H.O. Dämpfung bis $4 \mathrm{Bw}$. vereinzeltes Knacken, rechte Spitze steht tiefer. L.HO. und L.V.O. Giemen und Knacken. Tbc. nicht nachgewiesen.

24. VII. Fntfernang des Tamors in Lokalanästhesie (Lipom). Tuberkulinbehandlung vom 10. VII. (Dosis 0,1) bis 1. IX. (1,5), Gesamtdosis 12,0 ccm. Patient reagierte auf ' $\mathrm{T} b \mathrm{n}$. Res. bei den ersten Einspritzungen mit Stichreaktion. Bei der Dosis 0,3 trat eine Temperaturreaktion $(38,2)$ auf.

Befund bei der Entlassung: Pulmones: R.H O. geringe Schallverkürzung, vereinzeltes Knacken. Linke Lunge normaler Befund, wenig Husten und Auswurf, keine Nachtschweisse, subjektives Wohlbefinden, Gewicht 132 Pfd. Es ist also in diesem Falle eine wesentliche Besserung des Lungenbefundes festzustellen.

Nachuntersuchung: 20. II. 1914 subjektives Wohlbefinden, wenig Husten, keine Nachtschweisse.

Pulmones: R.H.O. geringe Schallverkürzung, Atemgeräusch verschärft, geringes Knisterrasseln im Exsp., rechte Spitze steht tiefer.

F a 11 13. Journal-Nr. 998, 13; männlich, 17 Jabre; behandelt vom 28. VIII. 13 bis 31 . I. 14. 
Anamnese: Ein Onkel an Tuberkulose gestorben, Patient hatte wiederholt Lungenentzündung, im Dezember 1912 hier wegen Bronchitis in Behandlung. Patient klagt jetzt über Stechen auf der. Brust, Mattigkeit, Husten, keine Nachtschweisse.

Status: 17jähriger junger Mensch, Ernährungszustand gut, Gewicht $131 \mathrm{Pfd}$. Supraklavikalargruben etwas eingesunken.

Pulmones: R.H. vom 5 Bw. ab Schallverkïrzung, auf der Höhe des Insp. pleuritisches Reiben mit durchklingendem entfernten Giemen. L.H.O. Schallverkürzung, abgeschwächtes Atmen. L.H.U. Giemen, Rasseln. Temperatur 38,5. Tbc. im Sputum und Urin nachgewiesen.

Diagnose: Tb. pulm., Bronchitis, Pleuritis.

Therapie: Tbn. Ros. Brustwickel, Liq. ammon. anisat

11. IX 13: Über beiden Oberlappen Rasselgeräusche, rechts stärker. R.V.O. verschärftes Insp. Husten, keine Nachtschweisse. 3. I. 14. R.H.O. vereinzeltes Knacken, ebenso rechts hinten unten. L.H.O. Knacken, nirgends Schallverkürzungen, keine Nachtschweisse, wenig Husten.

Tuberkulinkuren: 1. 27. VIII. bis 13. X. Anfangsdosis 0,1, Enddosis 1,5, Gesamtdosis $12 \mathrm{ccm}$. 2. 30. X. bis 14. XII. Dosierung wie bei 1. 3. 29. XII. bis 31. I. Anfangsdosis 0,1, Enddosis 1,2, Gesamtmenge 7,8. Im ganzen wurden $31,8 \mathrm{ccm}$ injiziert. Stichreaktionen traten bei den ersten Injektionen auf. Temperaturen nur in den ersten Tagen der Behandlung, später war Patient tieberfrei. Temperaturreaktionen wiederholt, jedoch 39,2 nicht überschreitend

Bakterienbefund: 19. VIII. Tbc. im Sputum und Urin positiv, vom 12. IX. in immer negativ. Gewicht bei der Aufnahme 131, Höchstgewicht 161 Pfd., Gewicht bei der Entlassung 153 Pfd. Die Sputummenge ging von 80 auf $25 \mathrm{ccm}$ herunter.

Entlassungsbefund: 31. I. Über der rechten Lunge vereinzeltes Rasse und Knacken, linke Spitze steht etwas tiefer. Perkut. normaler Befund. Subjektives Wohlbefinden, wenig Husten und Auswurf.

Nachuntersuchung: Patient fühlt sich wohl, ist seit Februar als Gärtner tätig. Nach starkem Husten R.H.O. vereinzeltes Knacken. Atemgeräusch unbestimmt. R.V. unter der Klav. verschärftes Insp. Der Fall zeigt eine ständige Besserung.

F a 11 14. Journal-Nr. 1022, 13; männlich, 24 Jahre; behandelt vem 25 . VIII. bis 20. X. 13 .

Anamnese: Mutter an Lungenentzündung, Vater an Tuberkulose gestorben. Patient erkrankte am 20. VIII. mit stechenden Schmerzen in beiden Seiten, fühlt sich sehr matt, will stark an Gewicht abgenommen haben; kein Husten, kein Auswurf, Nachtschweisse vorhanden.

Status: 24 jähriger kräftiger Mann, Thorax flach, Supraklavikulargruben eingesunken.

Pulmones: Perkut. normaler Befund.

Auskultat.: R.H.O. verlängertes Exsp., rereinzeltes Knacken. L.H.O. vereinzeltes Rasseln.

Diagnose: Tb. pulm.

Therapie: Tbu. Ros. Es wurden $12 \mathrm{ccm}$ Tbn. Ros. injiziert. Anfangsdosis 0,1, Enddosis 1,5. 26. IX.: R.H.O. and rechte Spitze verlängertes und verschärftes Exsp. Kein Rasseln und Knacken mehr. Nachtschweisse geschwunden. 22. X. R.H.O. wenig verlängertes Exsp. 
Keine Stichreaktion, keine Temperaturreaktionen. Tbc. nicht nachgewiesen, Gewichtszunahme 9 Pfd.

Nachuntersuchung: 11. III. 14. Subjektives Wohlbefinden, völlig normaler Lungenbefund, Patient ist als Fleischer tätig.

F a 11 15. Journal-Nr. 939, 13; männlich, 14 Jahre: behandelt vom 2. VIII. 13 bis $16 . \mathrm{X} .13$.

Anamnese: Vater an Tuberkulose gestorben, eine Schwester lungenkrank. Patient war hier wegen eines eingewachsenen Nagels in Behandlung, erkrankte am 5. VIII. mit Halsschmerzen, Husten, Nachtschweissen.

Status: 14 jähriger schwächlicher junger Mensch, Supraklavikulargruben eingesunken.

Pulmones: Über beiden Lungen bronchitische Geräusche, nach Abheilung der Bronchitis fand sich R.H.O. vereinzeltes Giemen, verschärftes Exsp. Tbc. nachgewiesen.

Diagnose: Tb. pulm. dextr. Es wurden $12 \mathrm{ccm}$ Tbn. Ros. injiziert. Anfangsdosis 0,1, Enddosis 1,5. 22. X. kein Husten, kein Auswarf, Nachtschweisse bisweilen. Subjektives Befinden gut.

Pulmones: R.H.O. verlängertes und verschärftes Exsp. Tbc. nicht mehr nachgewiesen. 'Temperatur nach Ablauf der Bronchitis normal, Temperaturreaktionen traten $2 \mathrm{mal}$, Stichreaktionen $4 \mathrm{mal}$ auf. Gewichtszuuabme 9 Pfd,

Nachuntersuchung: 9. März 1914. Patient klagt noch über etwas Husten und Auswurf, bisweilen Nachtschweisse, Untersuchung ergibt völlig normalen Lungenbefund.

F r 11 16. Journal-Nr. 1026, 13: männlich, 15 Jahre; behandelt vom 23 . VIII. bis $7 . \mathrm{X}, 13$

Anamnese: Patient kommt wegen heftiger Schmerzen in der rechten Unterbanchgegend, klagt ausserdem über Husten, Auswurf, Nachtschweissc. Befund: Es findet sich ein deutlicher Druckschmerz am Mc. Burney. Patient wird sofort appendektomiert; einige Tage nach der Operation Temperaturanstieg auf 40,0. Rechte Lunge gedämpft, Bronchialatmen.

Diagnose: Postoperative Pneumonie.

29. VIII. Temperaturabfall, noch starker Husten, im reicblichen Auswurf werden Tbc. nachgewiesen. 4. IX. R.H.O. Schallverkürzung bis zur Spina scapul., verłängertes Exsp, Knisterrasseln; rechte Spitze Schallverkürzung, feines Knistern.

Diagnose: 'Tb. pulm. dextr. 7. X. R.H.O. Schallverkürzung, verlängertes Exsp., kein Knistern. Es wurden $12 \mathrm{~cm}$ Tbn. Ros. injiziert. Anfangsdosis 0,1, Enddosis 1,5. Temperaturen nach Ablauf der akuten Erkrankungen normal. T'emperaturreaktionen und Stichreaktionen traten nicht auf. Tbc. zu Beginn der Behandlung nachgewiesen, später negativ, Gewichtszunahme 22 Pfd.

Nachuntersuchung: März 1914. Pulmones: R.H.O. geringe Schallverkürzung, verlängertes, verschärftes Exsp., vereinzeltes Rasselgeräusche, Husten, Auswurf noch vorbanden, subjektiv füblt Patient sich wohl. Objektıv ist eine geringe Verschlechterung gegenüber dem Entlassungsbefunde feststellbar.

Fall 17. Journal-Nr. 1006, 13; weiblich, 24 Jahre; behandelt vom 20 . VIII. bis 30. XII. 13.

Anamnese: Eine Schwester lungenkrank, seit 3 Wochen klagt Patientin über Kopfschmerzen, Mattigkeit und Herzklopfen. Husten, Auswurf und Nachtschweisse nicht vorhanden. 
Status: 24jähriges Mädchen, Ernährungszustand mässig, Hämoglobingehalt $75 \%$. Supraklavikulargruben eingesunken.

Pulmones: L.H.O. bis zur Spina scapul. Schallverkürzung, vereinzeltes Knäcken.

Diagnose: Tb. pulm. sin.

Therapie: Tbn. Ros.

Injektionen: am 28. VIII. 0,1, am 1. IX. 0,2, am 4. IX. 0,3. Patientin reagierte mit sehr starken Infiltraten, so stark, dass feuchte Verbände gemacht werden mussten. Die Temperaturreaktionen betrugen bei der sonst fieberfreien Patientin 37,6-38,4, 12-24. Stunden nach den Injektionen auftretend. Es wurde nun die Dosis auf 0,05 herabgesetzt. Da wieder ein Infiltrat entstand, wurde längere Zeit 0,025 intramuskulär gegeben. Es traten dann keine Infiltrate mehr auf. Die Dosis wurde dann langsam gesteigert bis 0,1 , dann immer um ein Dezigramm bis zur Enddosis 1,5. Tbn. Kos. wurde nun fast ohne Reaktionen ertragen. Gesamtdosis 18,1 $\mathrm{ccm}$.

Entlassungsbefund: L.H.O. geringe Schallverkürzung.

Auskultat.: Normaler Befund. Tbc. wurden nicht nachgewiesen. Gewichtszunahme $16 \mathrm{Pfd}$.

Nachuntersuchang: 12. II. Pat. fühlt sich wieder etwas matt, will $7 \mathrm{Pfd}$. abgenommen haben. Linke Spitze steht etwa $1 / 2$ Querfinger tiefer als die rechte, völlig normales Atemgeräusch über der ganzen Lunge, kein Husten, kein Auswurf.

F all 18. Journal-Nr. 822, 13; weiblich, 21 Jahre; behandelt vom 1. VII. bis $21 . \mathrm{X} .13$.

Anamnese: Pat. kommt wegen Magenbeschwerden zur Aufnahme.

Status: 21 jähriges Mädchen, Ernährungszustand mässig, Hämoglobin $65 \%$.

Pulmones: R H.O. geringe Schallverkürzang, Vesikuläratmen. L.H.O. verschärftes Atmen, vereinzeltes Knacken.

Abdomen: Druckschmerz zwischen Nabel und Proc. xiphoid. Säurewerte nach Probefrühstück: 20 freie $\mathrm{HCl}, 41$ Ges,-Acid.

Diagnose: Tb. pulm. sin.

Therapie: Tbn. Ros, Lenhartzkur einige Wochen. Es wurden $12 \mathrm{ccm}$ Tbn. Ros. injiziert. Anfangsdosis 0,1, Enddosis 1,5, Temperaturreaktionen wurden nicht beobachtet, bei der Dosis 0,7 trat ein Infiltrat auf. 21. X. L.H.O. etwas verschärftes Atemgeränsch, verlängertes Exsp., kein Husten, kein Auswurf, keine Nachtschweisse. Subjektives Wohlbefinden. Gewichtszunahme 21 Pfd.

Nachuntersuchung: März 1914. Patientin fühlt sich wohl, bisweilen Hnsten, Auswurf, keine Nachtschweisse.

Pulmones: L.H. in der Höhe des 7 Bw. verschärftes Atmen. L.H.O. geringe Verschärfung des Insp., im übrigen normaler Lungenbefund.

Fall 19. Journal-Nr. 1483, 13; männlich, 16 Jahre; behandelt vom 20. XII. 13 bis 2. II. 14 .

Anamnese: Vater, ein Bruder an Tuberkulose gestorben. Patient erkrankte vor 8 Tagen mit Stechen in der linken Brustseite; anfangs trockener Husten, später Auswurf und Nachtschweisse.

Status: 16 jähriger junger Mensch in gutem Ernährungszustand, Gewicht 103 Pfd. Thorax flach.

Pulmones: RechteSpitze verschärftes Insp., verlängertes und verschärftes Exsp.

Diagnose: $\mathrm{Tb}$. pulm. 
Therapie: Tbn. Ros. Auf die erste Injektion $(0,1)$ reagierte Pat, mit einer sehr starken Stichreaktion und einem Temperaturanstieg $(37,7)$, auch bei den 3 folgenden Injektionen $(0,2-0,4)$ traten starke Infiltrate und Temperaturreaktionen (bis 38,7) auf. Es wurde deshalb in der Dosis auf 0,05 und 0,025 heruntergegangen, bis Gewöhnung eintrat. Am Ende der Behandlung wurde 0,1 reaktionslos vertragen. Es wurden in 12 Injektionen $1,4 \mathrm{~cm}$ injiziert. Bei der Entlassung völlig normaler Langenbefund, etwas Husten und Answurf noch vorhanden, keine Nachtschweisse. Tbc. wurden nicht nachgewiesen. Gewichtszunahme 11 Pfd.

Nachuntersuchung: am 17. III. 14. Patient fühlt sich wobl, wenig Husten, kein Auswurf, keine Nachtschweisse. Befund.

Pulmones: R.H.O. geringe Verschärfung des Insp., im übrigen normaler

Fall 20. Journal-Nr. 1118, 13; weiblich, 21 Jahre; behandelt vom 2. I. 14 bis 23 . IV. 14.

Anamnese: Vater lungenkrank, ein Bruder an Tuberkulose gestorben. Pat. gibt an, am 23. XII. Blut ausgehustet zu haben, klagt über Mattigkeit und Husten, keine Nachtschweisse.

Status: Sehr schwächliches 21 jähriges Mädchen, Thorax flach.

Pulmones: Grenzen rechts weniger verschieblich als links. R.H.O. Schallverkürzung bis zur Spina scapulae, verschärftes Atmen, verlängertes Exsp. Rechte Spitze verschärftes Atemgeräusch.

Abdomen: Unterbaucbgegend auf Druck schmerzhaft, Uterus von derben Schwarten ummauert.

Diagnose: Tb. pulm. Parametritis gonorrhoica.

Es wurden $12 \mathrm{ccm}$ Tbn. Ros. injiziert. Anfangsdosis 0,1, Enddosis 1,5. 6. III. R.H.O. Schallverkürzıng bis zur Spina scapulae, verscbärftes pfeifendes Insp., feine Rasselgeräusche. Temperaturreaktionen traten nicht auf. Stichreaktionen wurden bei der Dosis 0,3 und 0,4 beobachtet. Gewichtszunahme $7 \mathrm{Pfd}$.

Befund am 4. IV. rechts H.O. geringe Schallverkürzung, normales Atemgeräusch, kein Husten, keine Nachtschweisse, morgens etwas Auswurf. Patientin fühlt sich wohl.

Betrachten wir die Änderung des Lungenbefundes bei diesen 20 Fällen, so ergibt sich in 6 Fällen eine Verschlechterung des Befundes (Fall 1-6). Bei 2 Fällen war der Befund annähernd gleichbleibend, während bei den übrigen 12 Patienten eine deutliche Besserung nachzuweisen war.

Bemerkenswert ist, dass es sich bei den 6 mit negativem Erfolg behandelten Fällen um vorgeschrittene Tuberkulose 2. und 3. Stadiums handelte.

Bei dem Fall 4 kann man sich des Eindruckes einer schädlichen Einwirkung des Tuberkuilin $\mathrm{R}$ osenbach nicht erwehren, nach drei Injektionen trat eine Verschlechterung des Lungenbefundes auf (Herdreaktion), von diesem Zeitpunkt an zeigt der Fall eine ständige Progredienz, Exitus letalis etwa 7 Monate nach Beginn der Behandlung. Auch bei den anderen 5 Fällen war ein ständiges Fortschreiten des Prozesses zu beobachten, obgleich die Fälle zum Teil lange in 
Behandlung waren und wiederholte Tuberkulinkuren vorgenommen wurden.

Bei 12 Patienten dagegen war eine günstige Beeinflussung zu erkennen. In fast allen Fällen war eine gute Gewichtszunahme, Besserung des Appetits und Hebung des Allgemeinbefindens festzustellen.

Unangenehme Nebenwirkungen der Injektionen in bezug auf das subjektive Befinden wurden nur in einem Fall beobachtet, der ambulant behandelt wurde. Es handelte sich um ein 12jähriges Schulmädchen, das nach den Injektionen über Mattigkeit, Kopfschmerz und Appetitlosigkeit klagte.

Husten und Nachtschweisse wurden durch die Tuberkulinbehandlung geringer, verschwanden in einigen Fällen gänzlich, auch die Sputummenge ging herunter.

Ganz entschieden günstig wirkt Tuberkulin Rosenbach auf die Temperaturen ein, es ist bei Fieber nicht kontraindiziert, sondern wirkt als Entfieberungsmittel; selbst bei einem Fall mit ausgedehntem Lungenbefunde wurde eine völlige Entfieberung erzielt.

Temperaturreaktionen (Erhebungen der Temperatur, die die Höchsttemperatur der vorausgegangenen Tage um mindestens 0,5 übersteigen) wurden bei 14 der 20 Fälle beobachtet.

Trat nach einer Injektion eine Fieberreaktion auf, so blieb man bei den folgenden Injektionen bei der gleichen Dosis, oder ging auf eine niedere Dosis zurück, es gelang dann leicht, Temperaturreaktionen zu vermeiden.

Sehr starke Reaktionen wurden bei den Fällen 17 und 19 beobachtet. Im ersten Falle gelang es durch ganz kleine Dosen $(0,025$ bis 0,05 ), die zum Teil intramuskulär gegeben wurden, Patienten an das Tuberkulin zu gewöhnen, so dass am Schlusse der Behandlung $1,5 \mathrm{ccm}$ reaktionslos vertragen, wurden.

Stichreaktionen in Form geröteter Infiltrate wurden wiederholt beobachtet, bei stärkerem Auftreten wurde ebenfalls in der Dosierung zurückgegangen. Bei dem eben erwähnten Falle 17 traten anfangs so starke Infiltrate auf, dass feuchte Verbände notwendig waren. Durch intramuskuläre Injektion kleiner Dosen gelang es auch hier, eine Gewöhnung zu erzielen und die Infiltrate zu vermeiden.

Geringe Herdreaktionen entgehen wohl in den meisten Fällen der Beobachtung, eine starke Herdreaktion zeigte der Fall 4.

Der Tuberkelbazillennachweis geschah mittels des Antiforminverfahrens; ein Verschwinden der Tuberkelbazillen nach der Behandlung mit Tuberkulin Rosenbach wurde bei 4 Fällen beobachtet. 
Auf Grund dieser wenigen Fälle ein abschliessendes Urteil über Tuberkulin Rosenbach zu fällen, ist natürlich unmöglich. Bei leichteren Erkrankungsformen hatten wir den Eindruck einer sehr günstigen Einwirkung, bei fortgeschrittenen Fällen ist die Anwendung nach unseren Erfahrungen nicht ratsam.

\section{Literatur.}

Friedrich J. Rosenbach, Ein neues Tuberkulin. Deutsche med. Wochenschr. 1910. Nr. 33 und 34.

H. Curschmann, Diagnostische und therapeatische Erfahrungen mit Tuberkulin Rosenbach. Verhandlung des deutschen Kongresses für innere Medizin. Wiesbaden 1912.

W. Kausch, Erfahrungen über Tuberkulin Rosenbach. Deatsche Med. Wochenschrift 1913. Nr. 6. 